\title{
A Rotating, Inhomogeneous Dust Interior for the BTZ Black Hole
}

\author{
Cenalo Vaz ${ }^{1}$ and K. R. Koehler ${ }^{2}$ \\ $R W C$ and Department of Physics, \\ University of Cincinnati \\ Cincinnati, Ohio 45221-0011, USA
}

\begin{abstract}
We present exact solutions describing rotating, inhomogeneous dust with generic initial data in $2+1$ dimensional AdS spacetime and show how they are smoothly matched to the Banados-Teitelboim-Zanelli (BTZ) solution in the exterior. The metrics, which are the rotational analogues of the $2+1$ dimensional LeMaitre-Tolman-Bondi (LTB) family, are described by their angular momentum and one additional constant which, together with the angular momentum, determines the energy density of the dust cloud. The weak energy condition gives a constraint on the angular momentum profile inside the cloud. Solutions can be stationary or time dependent, but only the time dependent solutions can be matched consistently to a BTZ exterior. No singularity is formed in either the stationary or the time dependent cases.
\end{abstract}

PACS Nos. 04.20.Jb, 04.60.Kz 04.70.Bw,

\section{INTRODUCTION}

Exact solutions in general relativity are valuable tools to explore the range of possible behaviors allowed by Einstein's field equations. They are also useful as comparisons for numerical or approximate analytical solutions. Among these, solutions describing the gravitational collapse of matter are of considerable interest.

During the collapse of a neutral body, one expects the spacetime outside it to relax into the spacetime of a Schwarzschild or, more generally, a Kerr black hole, while the matter itself undergoes continual collapse until a singularity, either covered or locally naked, of spacetime forms. However, the matter may also avoid the singular region entirely either halting in a self-sustaining object, dissipating or, when rotation is present, even passing through a white hole into another universe. To examine the range of possibilities exact solutions describing black hole interiors with various forms of (preferably rotating) matter are essential.

\footnotetext{
${ }^{1}$ email: Cenalo.Vaz@UC.Edu

2 email: Kenneth.Koehler@UC.Edu
} 
Spherical, non-rotating gravitational collapse has been studied with various forms of matter and the indication is that all the outcomes listed above may in principle be realized for regular initial data [1]. Critical behavior in the parameters, $p$, of the initial data have also been discovered [2, 3, 4, 5, 6]: above some critical value of the parameter a singularity is formed, below it the matter eventually dissipates and at it the solutions are oscillatory [7]. Of special interest is the fact that the critical values appear to be independent of spherical symmetry or the type of matter considered.

Compared to the spherical case, solutions with rotating matter smoothly matched to an exterior stationary vacuum are still poorly understood, although for some restricted classes of perfect fluids stationary solutions can be obtained [8, 9] by using a generalization of the solution generation procedure of [10, 11, 12]. The problem with obtaining general solutions is essentially in the complexity of the field equations.

A simpler setting for studying possible effects of rotation is the stationary solution of Banados, Teitelboim and Zannelli (BTZ) in 2+1 dimensions. The BTZ solution [13, 14] is given in stationary coordinates as

$$
d s^{2}=-f(r) d t^{2}+\frac{d r^{2}}{f}-r^{2}\left(d \phi-\frac{J^{2}}{r^{2}} d t\right)^{2},
$$

where

$$
f(r)=\Lambda r^{2}-M+\frac{J^{2}}{r^{2}}
$$

and $M$ and $2 J$ are conserved charges associated with the Killing vectors $\partial_{t}$ and $\partial_{\phi}$ They are interpreted as the mass and angular momentum of the black hole respectively (we take $8 \pi G=1)$. The solution can be obtained by non-standard identifications in AdS space [13, 15], and there is no curvature singularity. There are, however, two horizons at which the Lapse vanishes,

$$
r_{ \pm}^{2}=\frac{M}{2 \Lambda}\left(1 \pm \sqrt{1-\frac{4 \Lambda J^{2}}{M^{2}}}\right)
$$

an outer one at $r_{+}$and an inner (Cauchy) horizon at $r_{-}$. Closed time-like curves exist wihin the Cauchy horizon, but there is evidence [16] that the Cauchy horizon is unstable by mass inflation. It is of interest to know the fate of matter in the interior of the BTZ black hole and in particular if these features survive for reasonable initial data. For example the static BTZ black hole admits just one horizon and no curvature singularity, but when a collapsing and non-rotating dust interior is matched to the BTZ exterior a singularity does form at the center [17, 18]. Circularly symmetric null fluid collapse was examined by Husain [19], who found a variety of static limits, including the BTZ black hole but also other "hairy" black holes, depending on the equation of state. Self-similar perfect fluids were analyzed by Hirschmann et. al. [20] with interesting results showing both naked and covered singularities. Chan et. al [21, 22] found a rotating interior containing null dust and were able to show explicitly that mass inflation [23] causes the inner (Cauchy) horizon to become unstable. In this paper, we obtain exact, time dependent interiors representing rotating, time-like dust. Our solutions can be thought of as the rotational analogues of the LeMaitre-Tolman-Bondi [LTB] models in 2+1 dimensions. 
Another, somewhat different motivation for exact solutions describing gravitational collapse stems from the fact that quantum gravitational effects in the vicinity of gravitational singularities are of fundamental interest. In a couple of recent publications 24, 25, we developed a canonical, midisuperspace quantization of $2+1$ dimensional collapse without rotation with interesting results. Not only was it possible to obtain Hawking radiation, along with grey-body factors that result from relaxing the near horizon approximation, but canonical quantization also provided a new and transparent interpretation of the entropy of the BTZ black hole: the black hole is viewed as a single shell formed by the collapse of many dust shells, each of which occupies one of the energy levels available to the single final-state shell. The energy spectrum of the single shell in the final state as obtained from canonical quantization coincides with the spectrum obtained in [26], where it was proposed that because the asymptotic symmetry group of $2+1$ dimensional gravity with a negative cosmological constant is generated by two copies of the Virasoro algebra [27], its degrees of freedom should be described by two Conformal Field Theories (CFT's) at infinity. Explictly counting the microstates of the black hole in the canonical theory yields an Area Law which, when compared to the Bekenstein-Hawking entropy, connects the boundary term at the center with the central charge of the AdS/CFT approach. This connection is potentially far reaching, but it was established only in the absence of rotation. In order to fully compare the results of the AdS/CFT approach and the midisuperspace quantization program it is essential to quantize the system with rotation. To carry this out, exact interior solutions are required.

The plan of this paper is as follows. In Section II we obtain the equations of motion for rotating dust collapse in $2+1$ dimensions in the comoving frame. Einstein's equations determine both the velocity and the spatial gradient of the radius of collapsing shells, while also providing an integrability condition that relates the energy function and the angular momentum. We solve this integrability condition and examine the restrictions imposed by the weak energy condition on the angular momentum profile. In section III we solve the equations of motion for the dust shells. There are (critical) stationary solutions and timedependent solutions. The time-dependent solutions are oscillatory and, in both cases, the evolution does not lead to the formation of a singularity. In Section IV, we address the matching conditions. Because the interior solutions are obtained in a comoving (hence corotating) frame, it is not possible to directly match them to the BTZ solution as given in (3), which is given in stationary coordinates. Therefore, we first obtain the transformations that take the stationary BTZ system to a comoving system. The transformations are analogous to those obtained for non-rotating black holes in [28]. Our derivation is based on an exact solution of the geodesic equations, given in Appendix A. This allows us to directly compare the interior metric representing the dust filled spacetime with the exterior vacuum (BTZ) metric. We show that the stationary interiors cannot be matched to the BTZ vacuum unless the ADM mass is negative. They may, however, serve as $2+1$ dimensional cosmologies. We conclude in Section $\mathrm{V}$ with a few comments concerning the formation of trapped surfaces. Throughout, we follow the conventions of Weinberg [29]. 


\section{ROTATING DUST BALL IN 2+1 ADS GRAVITY}

We consider a general axially symmetric metric in $2+1$ dimensions of the form

$$
d s^{2}=e^{2 A}(d t-C d \varphi)^{2}-e^{2 B} d r^{2}-\left(R^{2}+C^{2} e^{2 A}\right) d \varphi^{2},
$$

where $A, B, C$ and $R$ are regarded as functions of $(t, r)$. The function $R(t, r)$ represents the curvature radius of the cloud. In the presence of a negative cosmological constant, Einstein's equations are of the form

$$
G_{\mu \nu}+\Lambda g_{\mu \nu}=-8 \pi G_{3} T_{\mu \nu}
$$

where $G_{3}$ is the $2+1$ dimensional Newton constant, $G_{\mu \nu}$ is the Einstein tensor, $-\Lambda(\Lambda>0)$ is the cosmological constant and $T_{\mu \nu}$ is the stress energy tensor (in what follows we set $8 \pi G_{3}=1$ ). We take the metric in (四) to be sourced by dust, so

$$
T^{\mu \nu}=\varepsilon(t, r) U^{\mu} U^{\nu},
$$

where $\varepsilon(t, r)$ represents the energy density of the collapsing dust cloud.

\section{A. Comoving Frame}

In comoving coordinates, the only non-vanishing component of $T^{\mu \nu}$ is $T^{00}=\varepsilon(t, r)$. The spatial components of the conservation equations then imply that

$$
\begin{gathered}
e^{2(A-B)} A^{\prime}=0 \\
C \dot{A}+\dot{C}=0,
\end{gathered}
$$

where dots refer to a derivatives with respect to $t$ and primes to derivatives with respect to $r$. The first of (7) requires that $A=A(t)$ is independent of $r$ and the second may be solved in terms of one integration function of $r$,

$$
C(t, r)=K(r) e^{-A(t)}
$$

It is possible to gauge fix so that $A=0$ making the metric in (4) of the form

$$
d s^{2}=d t^{2}-2 K(r) d t d \varphi-e^{2 B(t, r)} d r^{2}-R^{2}(t, r) d \varphi^{2} .
$$

The metric describes rotating dust with angular velocity

$$
\Omega(t, r)=-\frac{K}{R^{2}}
$$

and two non-vanishing components, $\omega_{r \varphi}=-\omega_{\varphi r}=-K^{\prime}$, of the vorticity. We assume that both $K$ and $K^{\prime}$ are non-vanishing throughout the cloud except perhaps at the boundary. Uniform rotation occurs when $K^{\prime}=0$; it is straightforward to show that the only solution of time-like dust with uniform rotation is the vacuum (BTZ) solution (this appears not to be 
the case for null dust [21]). The time component of the conservation equations then reduces to

$$
\partial_{t}\left(\varepsilon e^{B} \sqrt{K^{2}+R^{2}}\right)=0
$$

Its solution determines the energy density of the dust,

$$
\varepsilon(t, r)=\frac{F^{\prime}(r) e^{-B}}{\sqrt{K^{2}+R^{2}}}
$$

in terms of the metric functions and one unknown integration function, $F(r)$, whose gradient is required to be positive by the weak energy condition.

\section{B. Field Equations}

With (9) we find that

$$
G_{t r}+\frac{G_{r \varphi}}{K}=0 \Rightarrow\left(K^{2}+R^{2}\right) \dot{B}-R \dot{R}=0
$$

which equation is solved by

$$
e^{2 B}=\frac{R^{2}+K^{2}}{W^{2}}
$$

where $W=W(r)$ is a function only of $r$, which we take to be greater than zero. ${ }^{3}$

The angular and radial components of Einstein's equations now give an expression for the acceleration,

$$
G^{\varphi \varphi}=\frac{G^{r r}}{W^{2}}=4 \Lambda\left(K^{4}+R^{4}\right)-W^{2} K^{\prime 2}+4 R^{3} \ddot{R}+4 K^{2}\left(2 \Lambda R^{2}+\dot{R}^{2}+R \ddot{R}\right)=0
$$

which may be integrated and its solution given in terms of one integration function $E=E(r)$ as

$$
\dot{R}^{2}=-\Lambda R^{2}+\left(E-2 \Lambda K^{2}\right)-\frac{4 \Lambda K^{4}+K^{\prime 2} W^{2}-4 K^{2} E}{4 R^{2}} .
$$

Inserting this solution into $G^{t \varphi}=0$ simplifies the equation to give a condition on the spatial gradient, $R^{\prime}(t, r)$,

$$
G^{t \varphi}=0 \Rightarrow R^{\prime}=R\left(-\frac{E K}{W^{2} K^{\prime}}+\frac{W^{\prime}}{2 W}+\frac{K^{\prime \prime}}{2 K^{\prime}}\right)+\frac{1}{R}\left(-\frac{E K^{3}}{W^{2} K^{\prime}}-\frac{1}{2} K K^{\prime}+\frac{K^{2} W^{\prime}}{2 W}+\frac{K^{2} K^{\prime \prime}}{2 K^{\prime}}\right) .
$$

3 One could work with a dimensionless label coordinate,

$$
\rho=\int \frac{d r}{W}
$$

but we have chosen to keep $r$ and hence $W(r)$ arbitrary. 
As both $\dot{R}$ and $R^{\prime}$ are determined by the field equations, there must be one integrability condition that enforces consistency. This is indeed the case and it is provided by the $(t, r)$ component of the field equations which gives a Riccati equation for $E$,

$$
G^{t r}=0 \Rightarrow 2 E^{2} K+W^{2} K^{\prime}\left(E-\Lambda K^{2}\right)^{\prime}-E W\left(W K^{\prime}\right)^{\prime}=0 .
$$

(This condition can also be obtained by equating appropriate derivatives of (16) and (17).) It can be viewed as prescribing $E$ in terms of the metric functions $W$ and $K$ up to a constant of integration. When $K^{\prime} \neq 0$, the equation can be solved by quadratures since one of its solutions is $E=\sqrt{\Lambda} W K^{\prime}$. The general solution turns out to be

$$
E=\sqrt{\Lambda} W K^{\prime} \operatorname{coth}\left(2 \sqrt{\Lambda} \int \frac{K}{W} d r+H_{0}\right),
$$

where $H_{0}$ is an arbitrary constant. So the particular solution we started with is the $H_{0} \rightarrow \infty$ limit of the general solution. The limit $H_{0} \rightarrow-\infty$ gives another particular solution, $E=$ $-\sqrt{\Lambda} W K^{\prime}$.

The time-time component of the equations determines the energy density of the dust ball. Directly comparing $G^{t t}=-\varepsilon(t, r)$ with (12) gives

$$
F^{\prime}(r)=-\frac{2 E^{2} K^{2}}{W^{3} K^{2}}+\frac{2 E+2 \Lambda K^{2}}{W}-\left[\frac{\left(W K^{\prime}\right)^{\prime}}{2 K^{\prime}}\right]^{\prime},
$$

which the weak energy condition requires to be positive. First viewing $F^{\prime}$ as a function of $E$ we conclude that $E$ must lie between the roots of the equation $F^{\prime}=0$, both of which must be real. Then taking into account (19), we find that this amounts to a fairly complicated constraint on the angular momentum profile $K$ and the value of $H_{0}$. Equation (20) can be formally integrated using the integrability condition in (18) and we obtain $F(r)$,

$$
F(r)=\frac{E K}{W K^{\prime}}-\frac{\left(W K^{\prime}\right)^{\prime}}{2 K^{\prime}}+\int^{r} \frac{E}{W} d r
$$

so long as $K^{\prime} \neq 0$. We will show in section IV that consistent matching requires $K^{\prime}$ to vanish at the boundary of the matter with the BTZ vacuum. As $K^{\prime}$ approaches zero, (18) gives

$$
E=\frac{W^{2} K^{\prime \prime}}{2 K}
$$

and only the last term in (21) survives in the limit. One can now explicitly verify that all the independent field equations are satisfied.

In summary, the solutions are completely determined by the angular momentum and one arbitrary constant. The weak energy condition provides a complicated relationship between the angular momentum, its derivatives and the constant $H_{0}$, constraining the shape of the angular momentum profile. In the following section we integrate the remaining equation (16) for $R(t, r)$. 


\section{EXACT SOLUTIONS}

The Ricci scalar depends linearly on the energy density, so a curvature singularity must form when $R^{2}+K^{2}=0$. The Kretschmann scalar also diverges only in this limit, therefore there is no good reason to terminate the spacetime at $R=0$ and one should be able to continue the solution into the region $-K^{2}<R^{2}<0$. This suggests that $R^{2}=0$ is just a coordinate singularity and that $R^{2}+K^{2}=0$ should be interpreted as a ring singularity. However, the azimuthal Killing vector $\xi=\partial_{\varphi}$ becomes timelike when $R^{2}<0$. Since its orbits must be periodically identified, closed time-like curves will exist in a neighborhood of the ring singularity.

We choose the negative square root in (16) (to describe collapse) and integrate the equation, thereby getting

$$
\frac{1}{2} \int \frac{d x}{\sqrt{-\Lambda x^{2}+A x+\frac{B}{4}}}=-t+\frac{Q(r)}{2 \sqrt{\Lambda}},
$$

where we have set $x=R^{2}$,

$$
A=E-2 \Lambda K^{2}, \quad B=4 \Lambda K^{4}+\left(W K^{\prime}\right)^{2}-4 E K^{2},
$$

and $Q(r)$ is an arbitrary function or $r$, which should be compatible with (17). By transforming to $y=x+K^{2}$, it becomes clear that real solutions exist only so long as $E \geq \sqrt{\Lambda}\left|W K^{\prime}\right|$, and we find ${ }^{4}$

$$
R^{2}+K^{2}=\frac{E}{2 \Lambda}-\frac{\sqrt{E^{2}-\Lambda W^{2} K^{\prime 2}}}{2 \Lambda} \sin (2 \sqrt{\Lambda} t-Q) .
$$

Thus $R^{2}+K^{2}$ is strictly positive at all times and no strong curvature singularity will form. The solution $E=\sqrt{\Lambda}\left|W K^{\prime}\right|$ of (18), obtained in the $H_{0} \rightarrow \infty$ limit, determines a stationary (time-independent) solution. In this case, the dust is in a self-sustaining distribution with vanishing radial velocity, but for the weak energy condition to hold it is necessary for

$$
K^{\prime}>\frac{1}{2 \sqrt{\Lambda}}\left[\frac{\left(W K^{\prime}\right)^{\prime}}{2 K^{\prime}}\right]^{\prime}
$$

to be verified throughout the dust cloud.

For all finite values of $H_{0}$ the solutions are oscillatory. Equation (17) may be satisfied only if $Q(r)$ is an arbitrary constant, $Q_{0}$. This constant then determines the physical radius of any shell at the initial time, say $t=0$

$$
R^{2}(0, r)=\frac{E-2 \Lambda K^{2}}{2 \Lambda}+\frac{\sqrt{E^{2}-\Lambda W^{2} K^{\prime 2}}}{2 \Lambda} \sin \left(Q_{0}\right)
$$

and reflects a limited freedom in our choice of initial scaling, i.e., the shell radius $R(t, r)$ at the initial time. For instance, if we take $Q_{0}=0$ and use (27) to reexpress the function $F(r)$ in terms of the initial energy density profile, we find

$$
F(r)=\left.\int^{r} \varepsilon\left(0, r^{\prime}\right) \frac{K^{2}+R^{2}}{W}\right|_{t=0, r^{\prime}} d r^{\prime}=\frac{1}{2 \Lambda} \int^{r} \varepsilon\left(0, r^{\prime}\right) \frac{E\left(r^{\prime}\right)}{W\left(r^{\prime}\right)} d r^{\prime}
$$

\footnotetext{
4 This is also indicated by the solution (19) of the integrability condition (18).
} 
Furthermore, the initial radial velocity profile is obtained from (16),

$$
v_{0}^{2}(r)=\dot{R}^{2}(0, r)=\frac{E^{2}-\Lambda W^{2} K^{\prime 2}}{2\left(E-2 \Lambda K^{2}\right)} .
$$

Thus, if the dust cloud begins with zero initial radial velocity then $E=\sqrt{\Lambda} W K^{\prime}$ and the dust continues in stationary flow. For a general $E$ (compatible with the positive energy condition),

$$
R^{2}(t, r)=\frac{E-2 \Lambda K^{2}}{2 \Lambda}\left[1-\frac{\sqrt{2} v_{0}}{\sqrt{E-2 \Lambda K^{2}}} \sin (2 \sqrt{\Lambda} t)\right]
$$

reexpresses the solution (25) in terms of the initial velocity profile, $v_{0}(r)$.

\section{MATCHING TO THE BTZ EXTERIOR}

In the previous section we obtained collapsing dust solutions with rotation in the comoving system. For the solutions to represent the interior of a black hole, they should be shown to go over smoothly to a stationary exterior vacuum spacetime. In $2+1$ dimensions with

a negative cosmological constant, this is given by the BTZ solution in (3), which we write here as

$$
d s^{2}=f(\widetilde{R}) d T^{2}-\frac{d \widetilde{R}^{2}}{f(\widetilde{R})}-\widetilde{R}^{2}\left(d \phi-\frac{J}{\widetilde{R}^{2}} d T\right)^{2}
$$

where

$$
f(\widetilde{R})=\Lambda \widetilde{R}^{2}-M+\frac{J^{2}}{\widetilde{R}^{2}}
$$

T, $\widetilde{R}$ and $\phi$ are stationary coordinates, and it is not possible to directly compare the angular parts of the comoving dust solutions as is usually done in the absence of rotation. Instead we first use the solutions of the geodesic equations to set up a comoving coordinate system for the BTZ metric and then compare the interior and exterior in the usual way.

\section{A. Comoving Coordinates for the BTZ vacuum}

The equations for time-like geodesics of the BTZ space-time can be integrated exactly in terms of two constants of the motion, $P$ and $L$, representing the energy and angular momentum of the orbits respectively [30]. For our purposes we need only the velocities (see Appendix A). With proper time as affine parameter, they may be expressed as

$$
\begin{aligned}
U^{T} & =\frac{1}{\sqrt{f}} \cosh \eta, \\
U^{R} & =\sqrt{f} \sinh \eta \cos \alpha, \\
U^{\phi} & =\frac{1}{R} \sinh \eta \sin \alpha+\frac{J}{\widetilde{R}^{2} \sqrt{f}} \cosh \eta
\end{aligned}
$$


where

$$
\begin{aligned}
& \cosh \eta=\sqrt{\frac{P}{f}}-\frac{J L}{\widetilde{R}^{2} \sqrt{f}}, \\
& \sin \alpha=\frac{L}{\widetilde{R} \sinh \eta} .
\end{aligned}
$$

Consider a system of coordinates $(t, r, \varphi)$ that are comoving with respect to these orbits; in this system, $U^{t}=1$ and $U^{\varphi}=U^{r}=0$. Assuming that the transformation between the canonical BTZ coordinates and this comoving system depends only on $(t, r)$, we write

$$
\left(\begin{array}{c}
d T \\
d \widetilde{R} \\
d \phi
\end{array}\right)=\left(\begin{array}{ccc}
\frac{\cosh \eta}{\sqrt{f}} & T^{\prime} & 0 \\
\sqrt{f} \sinh \eta \cos \alpha & \widetilde{R}^{\prime} & 0 \\
\frac{1}{R} \sinh \eta \sin \alpha+\frac{J}{\widetilde{R}^{2} \sqrt{f}} \cosh \eta & \phi^{\prime} & 1
\end{array}\right)\left(\begin{array}{c}
d t \\
d r \\
d \varphi
\end{array}\right)
$$

Because $P$ and $L$ are constant along geodesics, they may be viewed as functions of $r$. There is therefore some freedom in our choice of the comoving coordinates. We may fix this freedom by imposing coordinate conditions in such a way as to obtain the simplest form for the BTZ metric in the comoving system. For example, requiring $g_{t r}=0$ we find

$$
T^{\prime}=\frac{\widetilde{R}^{\prime}}{f} \tanh \eta \cos \alpha,
$$

but we must verify that the condition is compatible with the integrability of the function $T(t, r)$. From (36),

$$
T(t, r)=h(t)+\int \frac{d \widetilde{R}}{f} \tanh \eta \cos \alpha
$$

and therefore

$$
\dot{T}=\dot{h}+\frac{\dot{\widetilde{R}}}{f} \tanh \eta \cos \alpha \Rightarrow \frac{\cosh \eta}{\sqrt{f}}=\dot{h}+\frac{\sinh ^{2} \eta \cos ^{2} \alpha}{\sqrt{f} \cosh \eta},
$$

where we have used (35). Inserting $\eta$ and $\alpha$ from the solutions in (34) we find that $\dot{h}(t)$ is independent of $r$ if and only if $P(r)=1$ and $L(r)=-J$. This gives $\dot{h}=1$ and therefore

$$
t=T-\int \frac{d \widetilde{R}}{f} \tanh \eta \cos \alpha .
$$

This choice of $P$ and $L$ also ensures that

$$
\phi^{\prime}=\frac{J \widetilde{R}^{\prime}}{\widetilde{R}^{2} f} \tanh \eta \cos \alpha
$$

is compatible with the integrability of $\phi$, for we have

$$
\phi=\varphi+g(t)+\int \frac{J d \widetilde{R}}{\widetilde{R}^{2} f} \tanh \eta \cos \alpha \Rightarrow \dot{\phi}=\dot{g}+\frac{J \dot{\widetilde{R}}}{\widetilde{R}^{2} f} \tanh \eta \cos \alpha
$$


and using (35) together with (34) we easily determine $\dot{g} \equiv 0$. Thus

$$
\varphi=\phi-\int \frac{J d \widetilde{R}}{\widetilde{R}^{2} f} \tanh \eta \cos \alpha .
$$

With (36) and (40), the transformation from stationary to comoving coordinates becomes

$$
\left(\begin{array}{c}
d T \\
d \widetilde{R} \\
d \phi
\end{array}\right)=\left(\begin{array}{ccc}
\frac{\cosh \eta}{\sqrt{f}} & \frac{\widetilde{R}^{\prime}}{f} \tanh \eta \cos \alpha & 0 \\
\sqrt{f} \sinh \eta \cos \alpha & \widetilde{R}^{\prime} & 0 \\
\frac{1}{R} \sinh \eta \sin \alpha+\frac{J}{\widetilde{R}^{2} \sqrt{f}} \cosh \eta & \frac{J \widetilde{R}^{\prime}}{\widetilde{R}^{2} f} \tanh \eta \cos \alpha & 1
\end{array}\right)\left(\begin{array}{c}
d t \\
d r \\
d \varphi
\end{array}\right)
$$

and the BTZ metric can be expressed in comoving coordinates with the line element

$$
d s^{2}=d t^{2}+2 J d t d \varphi-\frac{R^{\prime 2}}{1+J^{2} / \widetilde{R}^{2}} d r^{2}-\widetilde{R}^{2} d \varphi^{2}
$$

Note that

$$
\dot{R}^{2}=f \sinh ^{2} \eta \cos ^{2} \alpha=-\Lambda \widetilde{R}^{2}+\left(1+M-\Lambda J^{2}\right)+\frac{J^{2}(1+M)}{\widetilde{R}^{2}}
$$

has the same structure as (16), with constant coefficients determined by $M$ and $J$. Its solution can be given as

$$
R^{2}(t, r)=\frac{1+M-\Lambda J^{2}}{2 \Lambda}-\frac{1+M+\Lambda J^{2}}{2 \Lambda} \sin [2 \sqrt{\Lambda} t-Q(r)]
$$

where $Q(r)$ remains arbitrary. It will be seen that $R^{2}(t, r)$ is bounded from above but not from below. Thus the comoving coordinates cover only a portion of the spacetime. This happens because there exists a radial upper bound for the geodesics of massive particles [30]. Nevertheless, the upper bound for $R(t, r)$ is larger than the radius of the outer horizon and (44) is sufficient for verifying the matching conditions between the interior and the exterior spacetimes.

\section{B. Matching Conditions}

Direct comparison between the first fundamental forms describing the interior in (4) and the exterior in (44) on the boundary $r=r_{b}$, shows that one may identify the coordinates

$t$ and $\varphi$ along with $K\left(r_{b}\right)=K_{b}=-J$ and the physical radius $R\left(t, r_{b}\right)=\widetilde{R}\left(t, r_{b}\right) \stackrel{\text { def }}{=} R_{b}(t)$. Comparing the expressions for the radius function, we find that $K_{b}^{\prime}=K^{\prime}\left(r_{b}\right)=0$,

$$
1+M+\Lambda J^{2}=E_{b}
$$

and $Q\left(r_{b}\right)=Q_{0}=0$. Because the vorticity at the boundary is vanishing, there is an infinite discontinuity in the density $F^{\prime}(r)$ there, but $F$ itself remains continuous across the boundary. 
We must also ensure that the second fundamental forms match. The second fundamental form of the boundary surface in the interior has three non-vanishing components, viz.,

$$
K_{t \varphi}^{\mathrm{in}}=K_{\varphi t}^{\mathrm{in}}=\left.\frac{W K^{\prime}}{2 \sqrt{K^{2}+R^{2}}}\right|_{r_{b}}, \quad K_{\varphi \varphi}^{\mathrm{in}}=\left.\frac{W R R^{\prime}}{\sqrt{K^{2}+R^{2}}}\right|_{r_{b}},
$$

and in the exterior there is only one non-vanishing component,

$$
K_{\varphi \varphi}^{\text {out }}=\sqrt{J^{2}+R_{b}^{2}}
$$

Clearly, $K_{b}^{\prime}=0$ as before and

$$
R_{b}^{\prime}=\frac{J^{2}}{W_{b} R_{b}}+\frac{R_{b}}{W_{b}}
$$

This is guaranteed in the limit as $r \rightarrow r_{b}$ if $E_{b}=W_{b}^{2} K_{b}^{\prime \prime} / 2 K_{b}$ which, as shown in (22) solves the integrability condition (18) with $K_{b}^{\prime}=0$. We conclude that while the vorticity must vanish, $K^{\prime \prime}(r)$ cannot vanish at the boundary and moreover that $K_{b}^{\prime \prime} / K_{b}>0$ when the BTZ mass is positive.

On the other hand we had obtained stationary interiors when $E$ takes the critical value $E=\sqrt{\Lambda} W K^{\prime}$. Evidently, then $E_{b}=\sqrt{\Lambda} W_{b} K_{b}^{\prime}=0$ for the stationary interiors and, by (47), these metrics cannot be consistently matched to the BTZ vaccuum except when the ADM mass is negative. They may, however, serve as inhomogeneous, rotating $2+1$ dimensional cosmologies.

\section{CONCLUDING REMARKS}

Much can be said about the nature of the sigularity formed in gravitational collapse by examining the expansion, $\Theta$, of a congruence of twist free null geodesics [31]. However, if one is interested only in the trapped surfaces $(\Theta=0)$ there is a simpler but equivalent approach which we now briefly illustrate for the vacuum given in the time dependent form of (44). Consider null geodesics in this spacetime. The existence of an azimuthal Killing vector field, $\xi=\partial_{\varphi}$, implies a locally conserved quantity

$$
\xi_{\mu} U^{\mu}=g_{\varphi t} U^{t}+g_{\varphi \varphi} U^{\varphi}=L
$$

where $U^{\mu}=d x^{\mu} / d \lambda$ is tangent to a geodesic and $L$ may be interpreted as the angular momentum carried by it. Therefore, according to (51), a congruence of null geodesics carrying zero angular momentum (such a congruence is twist free and hypersurface forming) satisfies

$$
U^{\varphi}=\frac{J}{R^{2}} U^{t}
$$

so the null conditon,

$$
\left(1+\frac{J^{2}}{R^{2}}\right) U^{t^{2}}-\frac{R^{\prime 2}}{1+J^{2} / R^{2}} U^{r 2}-R^{2}\left(U^{\varphi}-\frac{J}{R^{2}} U^{t}\right)^{2}=0
$$


requires that along these geodesics

$$
\frac{d t_{n}}{d r}= \pm \frac{R^{\prime}}{1+J^{2} / R^{2}}
$$

where the positive sign refers to outgoing geodesics and the negative sign to infalling ones. The radius along an outgoing null ray is then given by $R_{n}=R\left(t_{n}(r), r\right)$, where $t_{n}(r)$ is the integral curve of (54), and the horizons are determined as the extrema of $R_{n}$,

$$
\frac{d R_{n}}{d r}=0=\dot{R}_{n} \frac{d t_{n}}{d r}+R_{n}^{\prime}
$$

Substituting (54) for outgoing radial geodesics and using (45), we find the condition

$$
R_{n}^{\prime}\left(\frac{\dot{R}_{n}}{1+J^{2} / R_{n}^{2}}+1\right)=R_{n}^{\prime}\left(-\frac{\sqrt{-\Lambda R_{n}^{2}+1+M-\Lambda J^{2}+\frac{J^{2}(1+M)}{R_{n}^{2}}}}{1+J^{2} / R_{n}^{2}}+1\right)=0 .
$$

Assuming that $R_{n}^{\prime} \neq 0$, the condition becomes

$$
-\Lambda R_{n}^{2}+1+M-\Lambda J^{2}+\frac{J^{2}(1+M)}{R_{n}^{2}}=\left(1+\frac{J^{2}}{R_{n}^{2}}\right)^{2}
$$

which admits three real solutions (provided that $M>4 \Lambda J^{2}$ ), two of which are positive,

$$
R_{n}^{ \pm 2}=\frac{M}{2 \Lambda}\left[1 \pm \sqrt{1-\frac{4 \Lambda J^{2}}{M}}\right]
$$

These will be recognized as the inner and outer horizons of the BTZ black hole, as determined in the introduction from the vanishing of the Lapse function in the stationary frame. The third root, $R_{n}^{2}=-J^{2}$, may be ignored as there is no reason to extend the vacuum solution to negative values of $R^{2}[13]$.

We may apply the same reasoning to determine the formation of an apparent horizon in the dust filled region. With $U^{\varphi}=-K / R^{2}$, the null condition requires that along these twist free null geodesics

$$
\frac{d t_{n}}{d r}= \pm \frac{\sqrt{R_{n}^{2}+K^{2}}}{W}
$$

The turning points of an outgoing ray are then given by

$$
0=\frac{d R_{n}}{d r}=\left(\frac{\dot{R}_{n} \sqrt{R_{n}^{2}+K^{2}}}{W}+R_{n}^{\prime}\right)
$$

and thus we find that the condition for the formation of an apparent horizon is

$$
-\dot{R}_{n} \sqrt{R_{n}^{2}+K^{2}}=W R_{n}^{\prime}
$$

where $\dot{R}_{n}$ is given in (16) and $R_{n}^{\prime}$ in (17). 
For the stationary solutions, this is just the condition $R^{\prime}=0$, i.e., at shell crossings. For the time dependent solutions, the condition once again yields a cubic equation for

$R_{n}^{2}$ and therefore admits at least one real root. Unfortunately, while the equation itself is straightforward to solve, the solutions are difficult to analyze without making further assumptions on the shape of the angular momentum profile in the dust ball. This goes beyond the scope of the present work.

In this paper we have examined time-like dust collapse with rotation in $2+1$ dimensions. A simplification of Einstein's equations was achieved by going to the comoving coordinate system. We found that all solutions are determined by one function ( $K$, the angular momentum) and one arbitrary constant $\left(H_{0}\right)$, and we examined the limits on $K$ and $H_{0}$ imposed by the weak energy condition. We determined stationary as well as oscillatory, time dependent solutions, but no singularity formation. To match the solutions in the comoving system to the BTZ black hole, we found a comoving system for the black hole, explicity giving the transformations from the stationary to the comoving frames. We demonstrated that the time dependent solutions can be matched to a BTZ exterior with positive ADM mass provided that $K_{b}^{\prime \prime} / K_{b}>0$ at the boundary, but the stationary solutions cannot be so matched. Thus the stationary spacetimes may be thought of as $2+1$ dimensional, inhomogeneous cosmologies, but not as black hole interiors. We have also presented a simplified approach to addressing the issue of trapped surfaces, although the conditions imposed by Einstein's equations are not as transparent as they are in the case of circular collapse and we could recover little information from the solutions without specializing to particular cases. However, the solutions do not at first glance seem to make a good toy model for analytically examining critical behavior because there are not varied outcomes and so it is not clear how useful such specializations might prove.

The cases of zero and positive cosmological constant (dS) may also be addressed from our expressions, in the first case by taking the limit as $\Lambda \rightarrow 0$ and in the second case by analytic continuation.

\section{Acknowledgments}

We are grateful to Louis Witten and T.P. Singh for stimulating discussions and valuable comments.

\section{Appendix A}

In this appendix we obtain the geodesics given in (33) and (34) for the BTZ spacetime. The condition for being time-like,

$$
f U^{T^{2}}-f^{-1} U^{\widetilde{R}^{2}}-\widetilde{R}^{2}\left(U^{\phi}-\frac{J}{\widetilde{R}^{2}} U^{T}\right)^{2}=1,
$$

allows us to reexpress the four velocities in terms of a "boost" and a rotation according to,

$$
U^{T}=\frac{1}{\sqrt{f}} \cosh \eta
$$




$$
\begin{aligned}
U^{\widetilde{R}} & =\sqrt{f} \sinh \eta \cos \alpha \\
U^{\phi}-\frac{J}{2 \widetilde{R}^{2}} U^{T} & =\frac{1}{\widetilde{R}} \sinh \eta \sin \alpha
\end{aligned}
$$

The geodesic equations can be written as

$$
\begin{aligned}
& \frac{d U^{T}}{d s}+\frac{d \ln f}{d s} U^{T}-\frac{2 J}{f \widetilde{R}}\left(U^{\phi}-\frac{J}{\widetilde{R}^{2}} U^{T}\right) U^{\widetilde{R}}=0 \\
& \frac{d U^{\widetilde{R}}}{d s}+\frac{f^{\prime}}{2}\left(f U^{T^{2}}-\frac{U^{\widetilde{R}^{2}}}{f}\right)-\widetilde{R} f\left(U^{\phi^{2}}-\frac{J^{2} U^{T^{2}}}{\widetilde{R}^{4}}\right)=0 \\
& \frac{d U^{\phi}}{d s}-\frac{2 J^{2}}{f \widetilde{R}^{3}}\left(U^{\phi}-\frac{J}{\widetilde{R}^{2}} U^{T}\right) U^{\widetilde{R}}+\frac{2 U^{\widetilde{R}}}{\widetilde{R}}\left(U^{\phi}+\frac{J(\ln f)^{\prime}}{\widetilde{R}} U^{T}\right)=0
\end{aligned}
$$

Subtracting the first from the last, we arrive after some manipulation at the equation

$$
\frac{d}{d s}\left(U^{\phi}-\frac{J}{\widetilde{R}^{2}} U^{T}\right)+\frac{2}{\widetilde{R}}\left(U^{\phi}-\frac{J}{\widetilde{R}^{2}} U^{T}\right) U^{\widetilde{R}}=0
$$

which is solved by

$$
U^{\phi}-\frac{J}{\widetilde{R}^{2}} U^{T}=\frac{L}{\widetilde{R}^{2}},
$$

where $L$ is constant on geodesics. Inserting this into the equation for $U^{T}$, we find that it can be integrated to yield

$$
U^{T}=\frac{\sqrt{P}}{f}-\frac{J L}{f \widetilde{R}^{2}}
$$

where $P$ is also constant on geodesics. Combining this with (63)

$$
\cosh \eta=\sqrt{\frac{P}{f}}-\frac{J L}{\widetilde{R}^{2} \sqrt{f}}
$$

and, using (66) in (63),

$$
\sin \alpha=\frac{L}{\widetilde{R} \sinh \eta}
$$

[1] P. Yodzis, H.J. Seifert and H. Müller zum Hagen, Commun. Math. Phys. 34 (1973) 135;

Commun. Math. Phys. 37 (1974) 29;

D. M. Eardley and L. Smarr, Phys. Rev. D 19, (1979) 2239;

D. Christodoulou, Commun. Math. Phys. 93 (1984) 171;

R. P. A. C. Newman, Class. Quant. Grav. 3 (1986) 527;

B. Waugh and K. Lake, Phys. Rev. D 38 (1988) 1315;

V. Gorini, G. Grillo and M. Pelizza, Phys. Lett. A 135 (1989) 154;

A. Ori and T. Piran, Phys. Rev. D 42, 1068 (1990); 
G. Grillo, Class. Quantum Grav. 8 (1991) 739;

R.N. Henriksen and K. Patel, Gen. Rel. Gravn. 23 (1991) 527;

I. H. Dwivedi and S. Dixit, Prog. Theor. Phys. 85 (1991) 433;

P. Szekeres and V. Iyer, Phys. Rev. D 47, 4362 (1993);

P. S. Joshi and I.H. Dwivedi, Phys. Rev D47 (1993) 5357;

I. H. Dwivedi and P.S. Joshi, Comm. Math. Phys. 166 (1994) 117;

S. Jhingan, P.S. Joshi, T. P. Singh, Class. Quant. Grav. 13 (1996) 3057;

I. H. Dwivedi, P. S. Joshi, Class. Quant. Grav. 14 (1997) 1223;

L. Herrera, A. Di Prisco, J. L. Hernandez-Pastora and N. O. Santos, [gr-qc/9711002];

P. S. Joshi and I. H. Dwivedi, Class. Quant. Grav. 16 (1999) 41;

S. S. Deshingkar, I. H. Dwivedi and P. S. Joshi, Phys. Rev. D59 (1999) 044018; ibid Gen. Rel. Grav. 30 (1998) 1477;

S. Jhingan and G. Magli, Phys. Rev D61 (2000) 124006.

[2] D. Christodoulou, Commun. Math. Phys. 105, 337 (1986); 106, 587 (1986); 109, 591 (1987); 109, 613 (1987); Commun. Pure Appl. Math. XLIV, 339 (1991); XLVI, 1131 1993; Ann. Math. 140, 607 (1994).

[3] M. W. Choptuik, Phys. Rev. Lett. 70, 9 (1993).

[4] A. Abraham and C. R. Evans, Phys. Rev. Lett. 70 (1993) 2980.

[5] C. Gundlach, Adv. Theor. Math. Phys. 2 (1998) 1; ibid Phys. Rept. 376 (2003) 339-405.

[6] F. Pretorius, M. W. Choptuik, Phys.Rev. D 62 (2000) 124012.

[7] A. Wang, Braz. J. Phys. 31 (2001) 188.

[8] H. Stephani, J. Math. Phys. 29, 1650 (1988)

[9] D. Garfinkle, E.N. Glass, J.P. Krisch, Gen. Rel. Grav. 29 (1997) 467.

[10] J. Ehlers, Les theories relativistes de la gravitation (CNRS, Paris, 1959).

[11] B. K. Harrison, J. Math. Phys. 9, 1744 (1968).

[12] R. Geroch, J. Math. Phys. 12, 918 (1971).

[13] M. Banados, M. Henneaux, C. Teitelboim, J. Zanelli, Phys.Rev. D 48 (1993) 1506.

[14] M. Banados, C. Teitelboim, J. Zanelli, Phys. Rev. D 49 (1994) 975.

[15] C. Vaz and L. Witten, Phys. Lett. B 327 (1994) 29.

[16] A. Steif, Phys.Rev. D49 (1994) 585.

[17] S.F. Ross and R.B. Mann, Phys.Rev. D 47 (1993) 3319.

[18] S. Gutti, Class. Quant. Grav. 22 (2005) 3223.

[19] V. Husain, Phys.Rev. D 52 (1995) 6860; ibid Phys. Rev. D 53 (1996) 1759.

[20] E. W. Hirschmann, A. Wang, Y. Wu, Class. Quant. Grav. 21 (2004) 1791.

[21] J.S.F. Chan, K.C.K. Chan, R.B. Mann, Phys. Rev. D 54 (1996) 1535.

[22] J.S.F. Chan, R.B. Mann, [gr-qc/9411064].

[23] E. Poisson and W. Israel, Phys. Rev. Lett. 63, 16, (1989) 1663.

[24] C. Vaz, S. Gutti, C. Kiefer, T.P. Singh, Phys. Rev. D 76, (2007) 124021.

[25] C. Vaz, S. Gutti, C. Kiefer, T. P. Singh, L.C.R. Wijewardhana, Phys. Rev. D 77 (2008) 064021.

[26] A. Strominger, JHEP 9802 (1998) 009.

[27] J. D. Brown and M. Henneaux, Comm. Math. Phys. 104 (1986) 207. 
[28] L.D. Landau and E.M. Lifshitz, The Classical Theory of Fields Vol II, Course of Theoretical Physics Series (4 ${ }^{\text {th }}$ Edition), Butterworth Heinneman, 1980.

[29] S. Weinberg, Gravitation and Cosmology: Principles and Applications of the General Theory of Relativity, Wiley (1972).

[30] N. Cruz, C. Martnez, L. Peña, Class. Quant. Grav. 11 (1994) 2731.

[31] T.P. Singh, Phys.Rev. D 58 (1998) 024004. 